\title{
Patient and staff experiences of remote kidney healthcare: lessons learnt from COVID-19
}

\author{
Courtney J. Lightfoot ${ }^{1,2}$ (1) Thomas J. Wilkinson $^{1,3} \cdot$ Jared Palmer $^{4} \cdot$ Archontissa M. Kanavaki $^{1} \cdot$ Alice C. Smith $^{1,2}$
}

Received: 27 August 2021 / Accepted: 21 September 2021 / Published online: 6 October 2021

(C) Italian Society of Nephrology 2021

Keywords Chronic kidney disease $\cdot$ COVID- $19 \cdot$ Remote healthcare $\cdot$ Barriers $\cdot$ Facilitators

\section{Introduction}

During the COVID-19 pandemic, healthcare systems have rapidly adopted alternative models for delivering healthcare (e.g., remote service) to minimise the spread of disease [1]. Understanding the impact this has on clinical practice, including patients' access to and quality of care, is important [2]. We explored the views and experiences of both people living with kidney disease and kidney clinicians on remote healthcare delivered during the pandemic in the UK.

\section{Brief methods}

In a multicentre observational study, we surveyed people with kidney disease and clinicians about their views and experiences of remote kidney healthcare delivered during the COVID-19 pandemic. A total of 5249 (2483 nondialysis, and 2766 transplant) patients, and 1167 healthcare professionals across 11 sites in England, UK, were invited to take part. Potential participants were sent a link to the online survey. The survey was conceived in response to the COVID-19 pandemic and widespread implementation of remote healthcare. Data were obtained using Jisc Online Surveys (Bristol, UK) between August and December 2020. The study was granted ethical approval by the Leicester

Courtney J. Lightfoot

courtney.lightfoot@leicester.ac.uk

1 Leicester Kidney Lifestyle Team, Department of Health Sciences, University of Leicester, Leicester LE17RH, UK

2 Leicester NIHR Biomedical Research Centre, Leicester, UK

3 NIHR Applied Research Collaboration East Midlands, Leicester Diabetes Centre, Leicester, UK

4 University Hospitals of Leicester NHS Trust, Leicester, UK
Research Ethics Committee (18/EM/0117). All patients provided informed online consent and the study was conducted in accordance with the Declaration of Helsinki. Descriptive and frequency data are presented as mean (standard deviation (SD)) or number (percentage). Free text responses were analysed using thematic analysis [3].

\section{Results}

A total of 549 (10\%) patients completed the survey: $56 \%$ were male, the mean age was $60.7\left({ }_{\mathrm{SD}} 12.7\right)$ years, $55 \%$ were transplant recipients, with a mean current transplant age of $11.4\left(\mathrm{SD}^{34.5)}\right.$ years. Fifty-nine (5\%) clinicians completed the survey: $70 \%$ were female with a mean age of 43.7 years $\left({ }_{\mathrm{SD}} 11.4\right), 44 \%$ were nurses and 28\% were consultants or specialist registrars. Participant characteristics are detailed in Supplementary Material 1.

\section{Patient experiences}

Prior to the pandemic, 65 (12\%) patients had previously experienced remote care. Most patients (91\%) reported having had a routine appointment or consultation since the onset of the pandemic; $70 \%$ of these were conducted remotely (i.e., video or telephone), $10 \%$ were face-to-face, and $11 \%$ received both. In total, 64 (13\%) felt that their appointment did not meet their expectations and/or needs. Of the 444 patients who had a remote appointment, 430 (97\%) stated they would consider having another remote appointment.

\section{Clinician experiences}

Forty-five clinicians (76\%) reported using remote methods for patient consultations. Of those, $22(49 \%)$ reported using them for outpatient appointments, 7 (16\%) reported using remote methods to assess patient needs when they reported a health 
problem, and $16(36 \%)$ reported using them for both. The most commonly used remote method was telephone $(n=43,96 \%)$, followed by email $(n=25,56 \%)$, then video $(n=16,36 \%)$. Of those who used remote care methods, $36(80 \%)$ were likely to continue using them in the future. Many suggested that remote methods could support face-to-face appointments and be used on an alternative basis (i.e., between face-to-face appointments).

\section{Facilitators and barriers of remote care}

Strengths and facilitators of remote care identified included: (1) increased efficiency, (2) reduced burden (including less time, reduced travel), (3) adequate time to prepare, and (4) effective communication. Weaknesses and barriers of remote care identified included: (1) reduced ability to perform clinical assessment and concerns around assessment accuracy, (2) communication difficulties, (3) perceived lack of confidentiality and disclosure, and (4) delayed intervention.

Exemplar quotes of the barriers and facilitators can be found in Supplementary Material 2.

\section{Future use of remote care}

Remote care was perceived to have the opportunity to be used more routinely, to support face-to-face appointments, and for specific groups such as those with stable disease.
Exemplar quotes of the future use of remote care can be found in Supplementary Material 2.

\section{Discussion}

Our findings show that remote kidney healthcare was wellaccepted, with many proposing a hybrid approach to future care (e.g., combination or alternative face-to-face and remote appointments). The balance between face-to-face appointments and remote appointments is delicate, with the optimal frequency of real-life contact undetermined [4]. Although, (the use of) remote appointments may be part of future healthcare, they are not considered to be a replacement for face-to-face appointments [4]. Given that the increased use of technology in routine care is part of the NHS 5 year plan [5], it is imperative that changes are implemented to improve current healthcare models and systems to enable successful integration of telemedicine in routine clinical practice.

Changes in the patterns of delivering care may involve complex interactions and implementation of change methods. Based on our findings, we identified strategies for optimising remote kidney care that could be utilised to ensure high-quality care is delivered to people with kidney disease (Fig. 1).
Fig. 1. 10 lessons learnt from delivery remote care during COVID-19 pandemic: strategies to optimise the delivery of future remote kidney healthcare

\section{Ten lessons learnt: strategies to optimise remote kidney care}

1. Triaging patients to identify the type of consultation that is required - establish criteria to identify those who need a face-to-face appointment (this may change over time, and be dependent on kidney function and health status)

2. Review patients to determine their competence with remote methods, taking into consideration any potential communication barriers (i.e. language, hearing) and computer literacy

3. Offer video-based remote care methods to achieve the most from the clinical appointment from both healthcare professional and patient perspective

4. Complete basic training about the digital systems available and how they can be used

5. Develop and improve remote care communication skills to encourage disclosure, maintain rapport, deal with distress and de-escalate aggression remotely

6. Introduce change processes to enhance monitoring or to flag 'at risk' patients to prevent delayed intervention

7. Identify techniques to recognise and manage remote care fatigue of clinicians

8. Redesigning of services may be required to ensure hybrid of remote and face-to-face clinic appointments which enable patients to transition between the two depending on their needs and requirement

9. Ensure that appropriate digital systems are in place and are used in the best way possible

10. Ensure that there are quality improvement structures and review processes in place to assess whether remote care methods are appropriate and consistent with standards of care 


\section{Limitations}

Whilst our patient sample was large and diverse from multiple secondary care sites across England, it was not entirely representative of the UK kidney population due to the exclusion of those receiving dialysis. Our clinician sample size was smaller, but not unexpected due to the high clinical demands at the time of the survey distribution. Due to the pandemic, our survey was conducted online; however, online surveys may not be easily accessible to all, and as a result our sample may under-represent people who are not online (typically older, less affluent, and with limited formal education) [6]

\section{Conclusion}

There are lessons to be learnt from delivering remote kidney care during the pandemic and potential strategies for optimising the delivery of future remote kidney care. A shift in the delivery of kidney care may require services to be designed to accommodate a new kidney care model. Further work is needed to ensure that remote care provides the same level of care, and whether discrepancies in the quality of care are perceived or experienced.

Supplementary Information The online version contains supplementary material available at https://doi.org/10.1007/s40620-021-01175-y.

Acknowledgements This report is independent research supported by the National Institute for Health Research (NIHR) Applied Research Collaboration East Midland (ARC-EM) and Leicester Biomedical Research Centre (BRC). The views expressed are those of the author(s) and not necessarily those of the Stoneygate Trust, NHS, NIHR ARCEM, Leicester BRC or the Department of Health. We would like to acknowledge the numerous research facilitators at each site who contributed to the collection of data in this study and other researchers who assisted with data collection, in particular Daniel Nixon.

Author contributions All authors contributed to the research idea and study design. CJL, TJW, JP, and AMK contributed to the generation/ collection of data. CJL performed the statistical analysis, and TJW assisted with data analysis/interpretation. CJL drafted the manuscript, and all authors reviewed the manuscript. Each author contributed important intellectual content during manuscript revision and accepts responsibility for the overall work by ensuring that questions pertaining to the accuracy or integrity of any portion of the work are appropriately investigated and resolved.
Funding This research was funded by the National Institute of Health Research (NIHR) Applied Research Collaboration East Midland (ARCEM) and the Stoneygate Trust, and supported by the National Institute for Health Research Leicester Biomedical Research Centre (BRC).

\section{Declarations}

Conflicts of interest The authors declare that they have no potential conflicts of interest with respect to the research, authorship, and/or publication of this article.

Ethics approval Ethical approval was granted by the Leicester Research Ethics Committee (24/05/2018, ref: 18/EM/0117). The study was undertaken in accordance with the Declaration of Helsinki.

\section{References}

1. Monaghesh E, Hajizadeh A (2020) The role of telehealth during COVID-19 outbreak: a systematic review based on current evidence. BMC Public Health 20(1):1193. https://doi.org/10.1186/ s12889-020-09301-4

2. Hutchings R (2020) The impact of COVID-19 on the use of digital technology in the NHS. Briefing August 2020. https://www.nuffi eldtrust.org.uk/files/2020-08/the-impactof-covid-19-on-the-useof-digital-technology-in-the-nhs-web-2.pdf.

3. Braun V, Clarke V (2006) Using thematic analysis in psychology. Qual Res Psychol 3(2):77-101. https://doi.org/10.1191/14780 88706qp063oa

4. Haveman ME, Kleiss SF, Ma KF, Vos CG, Ünlü Ç, Schuurmann RCL, Bokkers RPH, Hermens HJ, De Vries JPM (2019) Telemedicine in patients with peripheral arterial disease: is it worth the effort? Expert Rev Med Devices 16(9):777-786. https://doi.org/ 10.1080/17434440.2019.1649595

5. Maruthappu M, Sood HS, Keogh B (2014) The NHS five year forward view: transforming care. Br J Gen Pract 64(629):635. https://doi.org/10.3399/bjgp14X682897

6. Donkin L, Glozier N (2012) Motivators and motivations to persist with online psychological interventions: a qualitative study of treatment completers. J Med Internet Res 14(3):e91. https://doi. org/10.2196/jmir.2100

Publisher's Note Springer Nature remains neutral with regard to jurisdictional claims in published maps and institutional affiliations. 